\title{
Validation du modèle d'intention d'utilisation du paiement mobile en contexte de pandémie de COVID-19
}

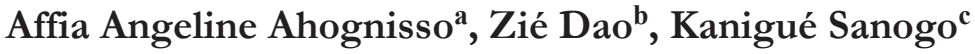

RÉSUMÉ. Le paiement mobile apparait comme une solution idéale dans cette période de lutte mondiale contre la pandémie de COVID-19. Cependant, plus de la moitié des Ivoiriens (soit 53,13 \%) continuent d'être réticents à l'utilisation du paiement mobile. Dans cette perspective, cette recherche explore les déterminants de l'utilisation du paiement mobile et de leur influence sur l'intention d'utilisation. L'influence de ces facteurs a été testée à l'aide d'un modèle d'équations structurelles sur un échantillon de 250 Ivoiriens. Les résultats montrent que la confiance et la facilité d'utilisation influencent positivement l'intention des Ivoiriens d'utiliser le paiement mobile. Quant à la sécurité perçue, une bonne perception de la sécurité n’a aucune influence sur l'intention d'utilisation, tandis qu'une mauvaise perception de la sécurité influence négativement l'intention d'utilisation.

\begin{abstract}
Mobile payment appears to be an ideal solution in this period of global fight against the COVID-19 pandemic. However, more than half of Ivorians (53.13\%) continue to be reluctant to use mobile payment. With this in mind, this research explores the determinants of the use of mobile payment and their influence on usage intention. The influence of these factors was tested using a structural equation model on a sample of 250 Ivorians. The results show that trust and ease of use positively influence the Ivorians' intention to use mobile payment. As for perceived security, a good perception of security has no influence on the intention to use, while a poor perception of security has a negative influence on the intention to use.
\end{abstract}

\section{Introduction}

Depuis la fin de 2019, une pandémie connue sous le nom de COVID-19 a été signalée pour la première fois à Wuhan, dans la province du Hubei, en Chine (Zhang et Ma, 2020). Depuis, la maladie n'a pas cessé de progresser dans le monde. À la fin du mois de mai, le rapport 132 du 31 mai 2020 de l'Organisation mondiale de la santé (OMS, 2020) indiquait un total de 5934936 cas d'infection à la maladie à coronavirus, dont 117551 cas en seulement 24 heures. À cette vitesse de propagation, la pandémie ne laisse personne indifférent (Tougan et Théwis, 2020). Elle a imposé au monde entier la mise en œuvre de mesures drastiques pour limiter la propagation du virus (Dube, 2020).
Parmi les mesures prises par de nombreux gouvernements pour lutter contre la propagation de la maladie, nous pouvons citer l'interdiction des rassemblements publics, la mise en place de distanciation sociale et, surtout, le confinement. Les populations sont contraintes de rester chez elles et de réduire au mieux le contact les unes avec les autres. En effet, selon l'OMS, le virus qui occasionne l'infection à la COVID-19 se transmet essentiellement par des gouttelettes produites lorsqu'une personne infectée tousse, éternue ou expire. Ces gouttelettes étant trop lourdes pour rester dans l'air, elles tombent rapidement au sol ou sur toute surface proche. Ainsi, toute personne à proximité d'une personne malade ou touchant une surface contaminée puis ses yeux, son nez et sa bouche pourrait être contaminée.

\footnotetext{
${ }^{a}$ D. Sc. Gestion, Université Félix-Houphouët-Boigny, Abidjan, Côte d'Ivoire, membre du Laboratoire de recherche en gestion des entreprises

b D. Sc. Gestion, Université Alassane Ouattara, Bouaké, Côte d’Ivoire, membre du Laboratoire de recherche en gestion des entreprises

c D. Sc. Gestion, Université Alassane Ouattara, Bouaké, Côte d’Ivoire, membre du Laboratoire de recherche en gestion des entreprises
} 
Cette situation très difficile a changé considérablement le mode de vie et le comportement d'achat de nombreuses personnes. Par ailleurs, on ne sait toujours pas quand la pandémie atteindra son apogée (Zhang et Ma, 2020). De ce fait, les populations devraient continuer, et ce, encore pour longtemps, à vivre sans certaines habitudes telles que les contacts physiques, les poignées de mains, les accolades, les attroupements, les transactions en face à face, etc. En raison de ce changement drastique de leurs habitudes, il est impératif de comprendre comment les populations s'adaptent pendant la pandémie.

À l'instar de tous les pays durement touchés, la Côte d'Ivoire, qui constitue le terrain de cette étude, continue de voir le nombre de cas infectés s'accroitre au sein de sa population. Ainsi, elle recommande, voire exige à celle-ci le strict respect des mesures dites de barrière à la propagation de la maladie.

Dans ce contexte difficile, une des options qui pourraient s'offrir aux consommateurs ivoiriens est l'adoption ou l'utilisation du paiement mobile. En effet, selon certaines recherches, le paiement mobile est l'un des moyens les plus sûrs pour les transactions à distance et sans contact (Jung, Kwon et Kim, 2020; Chaix et Torre, 2015; Chaix, 2013). Depuis plusieurs années, le paiement mobile connait une rapide évolution, surtout dans les économies en voie de développement ou faiblement bancarisées (Chaix et Torre, 2015). L'accroissement rapide de l'adoption des téléphones intelligents et les avancées technologiques dans les appareils mobiles sont régulièrement indexés pour être à la base de cette évolution. Ainsi, ces avancées permettent aux individus d'utiliser leur appareil mobile pour payer des biens et services ainsi que pour transférer de l'argent entre amis (Jung et ses collaborateurs, 2020). Les services de paiement mobile sont donc principalement caractérisés par le transfert d'argent et par le paiement sans contact. Ces caractéristiques pourraient s'avérer satisfaisantes au besoin de protection contre l'infection à la COVID-19.

En Côte d'Ivoire, la vulgarisation de l'argent mobile fait partie de la réglementation de la Banque Centrale des États d'Afrique de l'Ouest (BCEAO, 2015) incitant les institutions non bancaires à émettre de la monnaie électronique. Dès la mise en place de cette réglementation, cinq sociétés ont lancé un service d'argent mobile en Côte d'Ivoire :
Orange, MTN, Moov, CelPaid et Qash Services. Ces investissements, combinés à une initiative du gouvernement tendant à numériser les frais d'inscription scolaire dès 2014, sont des facteurs motivants à l'utilisation des services de paiement mobile. Pourtant, le rapport de l'Autorité de régulation des télécommunications de Côte d'Ivoire (ARTCI, 2019) du dernier trimestre de l'année 2019 indique que, sur un total de 37376603 abonnés aux services mobiles, seulement 17521108 sont utilisateurs, soit un taux d'utilisation de 46,87\%. Cela signifie que ces investissements n'ont visiblement pas suffi pour la majorité des Ivoiriens à se tourner vers le paiement mobile.

La réalisation de paiements est le principal cas d'utilisation de l'argent mobile en Côte d'Ivoire. Cependant, quels que soient les investissements des fournisseurs ou l'initiative du gouvernement, de nombreux travaux de recherche montrent que les abonnés de services de paiement mobile ne réalisent des paiements que lorsqu'ils sont convaincus de leur utilité (Baumüller, 2018; Deshmukh, Naware et Mumbai, 2014; Sridharan, Pramod et Sunitha, 2014; Yang, Lu, Gupta, Cao et Zhang, 2012).

Au regard de ce qui précède, il est évident que le paiement sans contact et à distance est utile dans un contexte d'interdiction des contacts physiques, des poignées de mains, des déplacements non indispensables, etc. Cependant, ce qui reste à savoir, c'est quelles sont les motivations des Ivoiriens à l'acceptation et/ou l'utilisation du paiement mobile?

Pour répondre à cette question, cette étude explore les déterminants de l'utilisation des services de paiement mobile, puis leur influence sur l'intention de les utiliser. Dans un premier temps, il s'agit d'identifier les facteurs qui déterminent l'utilisation de tels services. Dans un second temps, nous analysons l'influence de ces facteurs sur l'intention de la population ivoirienne d'utiliser le paiement mobile.

Dans cette période de lutte contre la pandémie de COVID-19, cette étude présente un grand intérêt à la fois pour les dirigeants d'entreprise et les potentiels utilisateurs du paiement mobile. En effet, elle permet aux fournisseurs de services de paiement mobile de connaitre les facteurs ou les caractéristiques qui motivent les Ivoiriens à leur utilisation. Quant à la population ivoirienne, cette étude indique un des moyens pour se protéger au mieux contre la pandémie de COVID-19 en évitant les contacts avec les autres. 


\section{Revue de littérature et développement des hypothèses de recherche}

La revue de littérature aborde les principaux concepts de la recherche ainsi que les relations qui existent entre eux.

\section{$1.1 \quad$ Confiance}

En sciences de gestion, le concept de confiance trouve ses origines dans la théorie des organisations. C'est une des méthodes fondamentales d'atteindre un optimum social et collectif. Précisément en marketing des services, l'étude de la confiance favorise le développement d'une relation durable entre le personnel en contact et le client (Gronroos, 1990; Perrien et Ricard, 1995). Selon Lagace et Gassenheimer (1991), la confiance est une attitude qui mène un partenaire à s'engager avec quelqu'un malgré une possibilité éventuelle de perte qui serait subie en raison du comportement de cette personne. Selon la définition de Moorman, Zaltman et Deshpande (1992), la confiance est comme une attente, une croyance, mais aussi une intention de comportement ou un comportement qui sous-entend la vulnérabilité et l'incertitude. La confiance étant conçue comme une croyance, elle consiste donc à compter sur une information reçue d'une autre personne à propos d'états incertains de l'environnement et de leurs conséquences au sein d'une situation de risque.

Dans la même veine, Ganesan (1994), Doney et Cannon (1997) ainsi que Ganesan et Hess (1997) associent à la confiance deux dimensions : 1) la crédibilité, qui est l'attribution au partenaire, la volonté de respecter ses promesses et 2) la bienveillance, qui représente les bonnes intentions du partenaire et son engagement à accorder la primauté à la satisfaction des besoins du client.

Rempel, Holmes et Zanna (1985) suggèrent plutôt une conception tridimensionnelle de la confiance. Premièrement, elle traduit la possibilité de prévoir le comportement à venir du partenaire en fonction de la consistance et de la stabilité de ses comportements passés. Deuxièmement, elle représente la possibilité de compter sur son partenaire en fonction des qualités, c'est-à-dire l'honnêteté, la franchise ou autres. Enfin, elle signifie le fait d'avoir foi en son partenaire,

Revue Organisations \& Territoires $\bullet$ Volume $30 \cdot \mathrm{N}^{\circ}{ }_{1} \cdot 2021$ sans que cette foi s'appuie sur des éléments tangibles liés à une expérience antérieure.

Gurviez et Korchia (2002) récapitulent les conceptions tridimensionnelles en définissant la confiance comme étant un ensemble de présomptions accumulées quant à la crédibilité, à l'intégrité ainsi qu'à la bienveillance du partenaire de l'échange. De son côté, Hess (1995) estime que le consommateur a confiance dans la marque quand il perçoit celle-ci comme étant fiable, honnête et altruiste. En fait, les dimensions proposées par Gurviez et Korchia (2002) sont analogues à celles identifiées par Hess (1995), car la crédibilité est l'équivalent de la fiabilité, l'intégrité est identique à l'honnêteté et, enfin, la bienveillance est synonyme d'altruisme.

Dans une perspective dichotomique de la confiance, Kramer et Tyler (1996) ainsi que Sirdeshmukh, Singh et Sabol (2002) considèrent que, d'une part, la confiance a lieu quand elle fait baisser les coûts des transactions. Elle conduit ainsi à l'évitement des coûts nécessaires afin de se protéger contre les comportements opportunistes et malhonnêtes des parties de la transaction. D'autre part, la confiance implique une prise de risque. Ainsi, elle implique un arbitrage cognitif entre les gains et les coûts qui lui sont subséquents.

En conséquence, nous formulons l'hypothèse suivante :

- La confiance influence positivement l'intention d'utilisation du paiement mobile en contexte de COVID-19 (hypothèse 1).

\subsection{Sécurité perçue}

Le concept de sécurité est perçu comme étant la protection de l'information ou des systèmes des menaces (Wang, Wang, Lin et Tang, 2003). Les menaces sont des circonstances, des conditions ou des évènements qui entrainent une peine économique aux données et ressources du système, sous forme de destruction, de modification des données, de blocage du service, de révélation, de modification et/ou fraude, abus et perte (Kalakota et Whinston, 1977).

Pousttchi et Schurig (2004) appréhendent la sécurité perçue comme étant le degré auquel une personne croit que l'utilisation d'une procédure de paiement mobile particulière serait sécurisée. 
Schierz, Schilke et Wirtz (2010) affirment que la sécurité perçue a une forte influence sur l'intention d'utilisation du paiement mobile.

Concernant le paiement mobile, les menaces peuvent être liées à la possibilité de piratage de comptes mobiles, à la fraude, à la qualité du réseau ou encore à des attaques à travers l'accès non autorisé aux comptes par une authentification fausse. Ainsi, un client qui croit que l'utilisation du paiement mobile est risquée adoptera probablement une attitude défavorable vis-à-vis du paiement mobile. Au contraire, un client qui croit que l'utilisation du paiement mobile est sécurisée adoptera une attitude favorable vis-à-vis du paiement mobile. En conséquence, nous formulons les deux hypothèses suivantes :

- La perception positive de sécurité influence positivement l'intention d'utilisation du paiement mobile en contexte de COVID-19 (hypothèse 2);

- La perception négative de sécurité influence négativement l'intention d'utilisation du paiement mobile en contexte de COV ID-19 (hypothèse 3).

\subsection{Facilité d'utilisation}

La facilité d'utilisation perçue est définie comme « le degré auquel une personne estime que l'utilisation d'un système particulier est exempte d'effort » (van der Heijden, 2002, p. 434, trad. libre). Cette explication découle de la définition du terme «facile»: être libre de toute difficulté ou sans effort. Les travaux de Venkatesh, Morris, Gordon, Davis et Fred (2003) démontrent que l'utilité ainsi que l'effort attendu (facilité d'utilisation) sont fortement reliés à l'intention d'utilisation. L'effort attendu est défini par Venkatesh et ses collaborateurs (2003) comme « le degré de facilité associée à l'utilisation du système» (p. 450, trad. libre) ou comme la facilité d'utilisation selon Davis (1989). Pour cet auteur, la facilité d'utilisation dépend de l'absence d'effort et de contrainte. Ainsi, la probabilité d'acceptation d'un système perçue comme étant facile à utiliser est élevée.

D'autres auteurs (Davis, Bagozzi et Warshaw, 1989; Adams, Nelson et Todd, 1992; Segars et Grover, 1993) démontrent, sur la base du modèle d'acceptation de la technologie (MAT), que la facilité d'utilisation perçue est liée à l'utilité perçue ainsi qu'à l'utilisation d'un système. Si Mallat, Rossi, Tuunainen et Öörni (2009) évoquent la mobilité,
Swanson (1982) considère l'accessibilité comme étant liée à la facilité d'utilisation perçue et affirme qu'ils sont en relation avec l'usage perçu. Quant à Shneiderman (1987) ainsi que Branscomb et Thomas (1984), ils attestent que la facilité d'utilisation affecte significativement l'usage ou non d'un système. En conséquence, nous proposons l'hypothèse suivante :

\section{- La facilité d'utilisation influence positivement l'intention d'utilisation du paiement mobile en contexte de COV ID-19 (hypothèse 4).}

\subsection{Intention d'utilisation}

Selon la théorie du modèle d'acceptation de la technologie (MAT), l'utilisation effective d'un système donné suscitera l'intention d'utilisation d'un individu qui est caractérisée simultanément par l'attitude de ce dernier avec l'utilisation d'un système (Ajzen et Fishbein, 1975). Quant à l'attitude c'est une disposition psychologique traduite par l'évaluation d'une entité particulière selon un degré de sentiments positifs sur la technologie.

Selon le MAT c'est l'attitude et l'utilité qui déterminent l'intention comportementale d'utiliser une technologie. L'analyse des travaux antérieurs met en exergue l'impact de l'utilité perçue sur l'intention. Cet impact est perceptible soit directement soit indirectement grâce à l'attitude. Selon Ajzen et Fishbein (1975), la probabilité que l'intention réelle se produise dépend du niveau de l'intention comportementale. Davis (1989), souligne que l'intention d'utilisation mesure la probabilité qu'une personne adopte une demande. Van der Heijden (2002), montre de façon empirique que l'attitude et l'intention expliquent et prédisent le comportement réel.

\section{Méthodologie}

Afin de tester les hypothèses de notre recherche, nous avons opté pour une étude empirique composée de deux phases: l'une exploratoire et l'autre confirmatoire. Le cadre méthodologique a été conçu autour des propositions faites par des auteurs tels que Gerbing et Anderson (1988) et Rossiter (2002). La méthodologie adoptée s'articule autour de deux points : nous présentons successivement la méthode de collecte, puis la mesure des variables. 


\subsection{Méthode de collecte}

Dans le cadre de la collecte de nos données, nous avons utilisé un questionnaire. Le questionnaire a été soumis à l'ensemble de l'échantillon de l'étude en face à face. Au total, nous avons interrogé près de 200 individus pour la phase exploratoire et nous avons choisi par convenance 250 individus pour la phase confirmatoire. Toutefois, nous avons pris le soin de diversifier l'échantillon. Ainsi, parmi les 250 personnes interrogées, on dénombre 126 hommes (50,4\%), ce qui signifie qu'il y a quasiment autant de femmes (49,6\%) répondantes. Par ailleurs, en ce qui concerne l'âge des répondants, on note la répartition suivante: des personnes âgées de 18 à 25 ans $(32 \%)$, de 26 à 35 ans $(38 \%)$, de 36 à 45 ans $(15,6 \%)$, de 46 à 55 ans $(10,6 \%)$ et de 56 ans et plus $(3,6 \%)$.

\subsection{Mesure des variables}

Le questionnaire soumis a été adapté de la littérature, notamment des travaux d'auteurs comme Sweeney et Soutar (2001), Ettis et Haddad (2014), Le Roux, Chandon et Strazzieri (1997) ainsi que Thompson, Higgins et Howell (1991). La sélection des échelles de mesure s'est faite à partir de leurs propriétés psychométriques (fiabilité, validité et stabilité).

Concernant la mesure de la confiance, la littérature révèle deux conceptualisations : une unidimensionnelle et l'autre multidimensionnelle. Dans le cadre de la présente recherche, nous avons opté pour la conceptualisation unidimensionnelle, compte tenu de l'orientation générale des items utilisés. Ainsi, avons-nous adapté cinq items des travaux de Le Roux et ses collaborateurs (1997) ainsi que de Lee (2005).

La sécurité perçue a été opérationnalisée à partir des travaux d'Ettis et Haddad (2014) et l'échelle de mesure utilisée comprend six items. La perception de la sécurité est appréhendée de deux façons : une catégorie d'individus estime que le paiement mobile est sécurisé, tandis que l'autre catégorie pense le contraire. L'ensemble des items mesure le risque lié à l'utilisation du paiement mobile.

La facilité d'utilisation représente une mesure de la perception des individus sur la facilité de l'acte ou de la tâche à accomplir dans l'utilisation du téléphone mobile. Dans notre contexte, ces tâches se résument au paiement. Afin de l'opérationnaliser, nous nous sommes appuyés sur les travaux de Venkatesh, Morris, Davis et Davis (2003), de Venkatesh, Thong et $\mathrm{Xu}$ (2012) ainsi que de Thompson et ses collègues (1991). Au total, six items nous ont permis d'appréhender la facilité d'utilisation.

Enfin, l'intention d'utilisation mesure la probabilité qu'une personne utilise le paiement mobile. Pour mesurer cette intention, nous nous sommes appuyés sur les travaux de Dabholkar et Bagozzi (2002), qui perçoivent l'intention comme un prédicateur du comportement d'usage. Cette variable permet de cerner l'intention et l'usage planifié du paiement mobile. L'échelle de mesure utilisée comporte sept items.

\section{Résultats}

Afin de tester les qualités psychométriques des variables de la présente recherche, nous avons, dans un premier temps, réalisé une analyse factorielle exploratoire, puis, dans un second temps, une analyse factorielle confirmatoire.

\subsection{L'analyse factorielle exploratoire}

L'analyse factorielle exploratoire s'est faite par le biais d'une analyse en composantes principales (ACP). Avant de passer à l'ACP, qui s'est faite par rotation varimax, nous nous sommes assuré que les données étaient factorisables (indice de KaiserMeyer-Olkin $(\mathrm{KMO})>0,5$; test de sphéricité de Bartlett significatif) (voir tableau 1). 


\begin{tabular}{|l|c|c|c|c|}
\hline Indices & Confiance & $\begin{array}{c}\text { Sécurité } \\
\text { perçue }\end{array}$ & $\begin{array}{c}\text { Facilité } \\
\text { d'utilisation }\end{array}$ & $\begin{array}{c}\text { Intention } \\
\text { d'utilisation }\end{array}$ \\
\hline Indice de Kaiser-Meyer-Olkin $(\mathrm{KMO})$ & 0,870 & 0,773 & 0,857 & 0,909 \\
\hline Test de sphéricité de Bartlett significatif & 0,000 & 0,000 & 0,000 & 0,000 \\
\hline Valeur propre & 3,332 & $3,071 / 1,077$ & 3,405 & 4,657 \\
\hline \% de variance expliquée & 66,649 & 69,140 & 68,102 & 66,534 \\
\hline Fiabilité $(\alpha$ de Cronbach) & 0,873 & $0,819 / 676$ & 0,881 & 0,912 \\
\hline
\end{tabular}

Tableau 1 - Résultats de l'analyse factorielle exploratoire

Source : Données terrain

La factorisation nous a permis de restituer $66,649 \%$ de la confiance, $69,140 \%$ de la sécurité perçue, $68,102 \%$ de la facilité d'utilisation et $66,534 \%$ de l'intention d'utilisation. Afin de tester la fiabilité des instruments de mesure, nous avons calculé les alphas de Cronbach; ceux-ci sont tous supérieurs à 0,6. Par conséquent, les instruments de mesure sont fiables.

L'analyse factorielle exploratoire effectuée a permis d'identifier une dimension de la confiance, deux dimensions de la sécurité perçue, une dimension de la facilité d'utilisation et une dimension de l'intention d'utilisation. Les structures factorielles identifiées à l'issue de l'ACP sont toutes conformes aux échelles existantes.

\subsection{L'analyse factorielle confirmatoire}

L'analyse factorielle confirmatoire a reposé sur une modélisation par équations structurelles. L'ensemble des traitements s'est fait à l'aide du logiciel Amos, ce qui nous a permis non seulement de tester la fiabilité, mais aussi la validité (convergente et discriminante) des échelles. L'ajustement des différentes mesures a été fait sur les données de la seconde collecte. Dans l'ensemble, les différentes mesures présentent de bonnes qualités d'ajustement (indices absolus, indices incrémentaux et indices de parcimonie).

\section{La confiance}

Pour la variable confiance (voir tableau 2), nous observons les données suivantes: RMSEA = 0,000; $\mathrm{CFI}=1,000 ; \mathrm{GFI}=0,996$ et le degré de liberté $\mathrm{khi}^{2}=0,943$. L'ensemble des indices satisfait aux exigences de la littérature.

\begin{tabular}{|l|l|c|c|}
\hline $\begin{array}{l}\text { Catégorie } \\
\text { d'indices }\end{array}$ & $\begin{array}{l}\text { Indices } \\
\text { retenus }\end{array}$ & $\begin{array}{c}\text { Valeurs de } \\
\text { référence }\end{array}$ & $\begin{array}{c}\text { Modèle issu } \\
\text { de l'AFE }\end{array}$ \\
\hline \multirow{4}{*}{ Indices absolus } & AGFI & 0,9 & 0,987 \\
\cline { 2 - 4 } & GFI & 0,9 & 0,996 \\
\cline { 2 - 4 } & SRMR & $<0,05$ & 0,011 \\
\cline { 2 - 4 } & RMSEA & 0,08 & 0,000 \\
\cline { 2 - 4 } & $P$ & $>0,05$ & 0,451 \\
\hline \multirow{2}{*}{$\begin{array}{l}\text { Indices } \\
\text { incrémentaux }\end{array}$} & CFI & 0,9 & 1,000 \\
\cline { 2 - 4 } $\begin{array}{l}\text { Indices de } \\
\text { parcimonie }\end{array}$ & Khi $2 /$ ddl & 0,9 & 0,995 \\
\cline { 2 - 4 } & CAIC & 5 & 0,943 \\
\hline
\end{tabular}

Tableau 2 - Résultats de l'analyse factorielle confirmatoire de la confiance Source : Données terrain 
La sécurité perçue

Concernant la variable sécurité perçue (voir tableau 3), les indices obtenus sont les suivants:
RMSEA $=0,075 ;$ CFI $=0,951$, GFI $=0,987$ et le degré de liberté $\mathrm{khi}^{2}=3,492$. Au regard des indices obtenus, le modèle s'ajuste bien aux données.

\begin{tabular}{|c|c|c|c|c|c|c|}
\hline $\begin{array}{l}\text { Catégorie } \\
\text { d'indices }\end{array}$ & $\begin{array}{l}\text { Indices } \\
\text { retenus }\end{array}$ & $\begin{array}{l}\text { Valeurs de } \\
\text { référence }\end{array}$ & $\begin{array}{l}\text { Modèle à } \\
\text { une } \\
\text { dimension }\end{array}$ & $\begin{array}{l}\text { Modèle } \\
\text { issu de } \\
\text { l'AFE }\end{array}$ & $\begin{array}{c}\text { Modèle } \\
\text { AFE sans } \\
\text { S4 }\end{array}$ & $\begin{array}{l}\text { Modèle de } \\
\text { second } \\
\text { ordre }\end{array}$ \\
\hline \multirow{5}{*}{$\begin{array}{l}\text { Indices } \\
\text { absolus }\end{array}$} & AGFI & 0,9 & 0,801 & 0,886 & 0,952 & 0,952 \\
\hline & GFI & 0,9 & 0,915 & 0,957 & 0,987 & 0,987 \\
\hline & SRMR & $<0,05$ & 0,096 & 0,053 & 0,025 & 0,025 \\
\hline & RMSEA & 0,08 & 0,162 & 0,118 & 0,075 & 0,075 \\
\hline & $P$ & $>0,05$ & 0,000 & 0,000 & 0,007 & 0,007 \\
\hline \multirow{2}{*}{$\begin{array}{l}\text { Indices } \\
\text { incrémentaux }\end{array}$} & CFI & 0,9 & 0,605 & 0,812 & 0,951 & 0,951 \\
\hline & NFI & 0,9 & 0,594 & 0,794 & 0,935 & 0,935 \\
\hline \multirow{2}{*}{$\begin{array}{l}\text { Indices de } \\
\text { parcimonie }\end{array}$} & $\mathrm{Khi}^{2} / \mathrm{ddl}$ & 5 & 12,497 & 7,136 & 3,492 & 3,492 \\
\hline & CAIC & $\begin{array}{c}<\text { au CAIC } \\
\text { du modèle } \\
\text { saturé }\end{array}$ & $\begin{array}{c}197,518 \\
(148,822)\end{array}$ & $\begin{array}{c}149,214 \\
(148,822)\end{array}$ & $\begin{array}{c}91,923 \\
(106,302)\end{array}$ & $\begin{array}{c}91,923 \\
(106,302)\end{array}$ \\
\hline
\end{tabular}

Tableau 3 - Résultats de l'analyse factorielle confirmatoire de la sécurité perçue Source : Données terrain

\section{La facilité d'utilisation}

Concernant la facilité d'utilisation (voir tableau 4), nous observons les données suivantes : RMSEA $=$
0,000; $\mathrm{CFI}=1,000 ; \mathrm{GFI}=0,998$ et le degré de liberté $\mathrm{khi}^{2}=0,890$. Nous pouvons donc conclure que le modèle s'ajuste bien aux données.

\begin{tabular}{|l|l|c|c|c|}
\hline $\begin{array}{l}\text { Catégorie } \\
\text { d'indices }\end{array}$ & $\begin{array}{l}\text { Indices } \\
\text { retenus }\end{array}$ & $\begin{array}{c}\text { Valeurs de } \\
\text { référence }\end{array}$ & $\begin{array}{c}\text { Modèle } \\
\text { issu de } \\
\text { 1'AFE }\end{array}$ & $\begin{array}{c}\text { Modèle } \\
\text { respécifié sans } \\
\text { FU5 }\end{array}$ \\
\hline \multirow{4}{*}{$\begin{array}{l}\text { Indices } \\
\text { absolus }\end{array}$} & AGFI & 0,9 & 0,942 & 0,990 \\
\cline { 2 - 5 } & GFI & 0,9 & 0,981 & 0,998 \\
\cline { 2 - 5 } & SRMR & $<0,05$ & 0,025 & 0,008 \\
\cline { 2 - 5 } & RMSEA & 0,08 & 0,086 & 0,000 \\
\cline { 2 - 5 } & $P$ & $>0,05$ & 0,001 & 0,410 \\
\hline \multirow{2}{*}{$\begin{array}{l}\text { Indices } \\
\text { incrémentaux }\end{array}$} & $\mathrm{CFI}$ & 0,9 & 0,912 & 1,000 \\
\cline { 2 - 5 } $\begin{array}{l}\text { Indices de } \\
\text { parcimonie }\end{array}$ & $\mathrm{Khi} / \mathrm{ddl}$ & 0,9 & 0,891 & 0,989 \\
\cline { 2 - 5 } & $\mathrm{CAIC}$ & 5 & 4,221 & 0,890 \\
\hline & & $\begin{array}{c}<\text { au CAIC du } \\
\text { modèle saturé }\end{array}$ & $\begin{array}{c}91,972 \\
(106,302)\end{array}$ & $\begin{array}{c}58,475 \\
(70,868)\end{array}$ \\
\hline
\end{tabular}

Tableau 4 - Résultats de l'analyse factorielle confirmatoire de la facilité d'utilisation Source : Donnée terrain 


\section{L'intention d'utilisation}

Pour ce qui est de l'intention d'utilisation (voir tableau 5), nous avons obtenu les indices suivants:
RMSEA $=0,073 ;$ CFI $=0,900 ;$ GFI $=0,977$ et le degré de liberté $\mathrm{khi}^{2}=3,322$. Les résultats montrent que le modèle retenu s'ajuste bien aux données.

\begin{tabular}{|l|l|c|c|c|}
\hline $\begin{array}{l}\text { Catégorie } \\
\text { d'indices }\end{array}$ & $\begin{array}{l}\text { Indices } \\
\text { retenus }\end{array}$ & $\begin{array}{c}\text { Valeurs de } \\
\text { référence }\end{array}$ & $\begin{array}{c}\text { Modèle } \\
\text { issu de } \\
\text { 1'AFE }\end{array}$ & $\begin{array}{c}\text { Modèle } \\
\text { respécifié sans } \\
\text { INT7 }\end{array}$ \\
\hline \multirow{4}{*}{$\begin{array}{l}\text { Indices } \\
\text { absolus }\end{array}$} & AGFI & 0,9 & 0,921 & 0,947 \\
\cline { 2 - 5 } & GFI & 0,9 & 0,961 & 0,977 \\
\cline { 2 - 5 } & SRMR & $<0,05$ & 0,032 & 0,021 \\
\cline { 2 - 5 } & RMSEA & 0,08 & 0,087 & 0,073 \\
\cline { 2 - 5 } & $P$ & $>0,05$ & 0,000 & 0,000 \\
\hline \multirow{2}{*}{$\begin{array}{l}\text { Indices } \\
\text { incrémentaux }\end{array}$} & CFI & 0,9 & 0,802 & 0,900 \\
\cline { 2 - 5 } $\begin{array}{l}\text { Indices de } \\
\text { parcimonie }\end{array}$ & Khi $2 /$ ddl & 0,9 & 0,763 & 0,865 \\
\cline { 2 - 5 } & CAIC & 5 & 4,325 & 3,322 \\
\hline & & $\begin{array}{c}<\text { au CAIC du } \\
\text { modèle saturé }\end{array}$ & $\begin{array}{c}159,767 \\
(198,430)\end{array}$ & 114,938 \\
& & & & \\
\hline
\end{tabular}

Tableau 5 - Résultats de l'analyse factorielle confirmatoire de l'intention d'utilisation Source : Données terrain

\section{La fiabilité et la validité des échelles de mesure}

La fiabilité et la validité des échelles de mesure utilisées ont été appréciées par le biais du rhô de Jöreskog et du rhô de validité convergente ou variance moyenne extraite. Tous ces indices sont obtenus par le truchement du modèle de mesure global (voir tableau 6).

\begin{tabular}{|l|l|c|c|c|}
\hline $\begin{array}{l}\text { Catégorie } \\
\text { d'indices }\end{array}$ & $\begin{array}{l}\text { Indices } \\
\text { retenus }\end{array}$ & $\begin{array}{c}\text { Valeurs de } \\
\text { référence }\end{array}$ & $\begin{array}{c}\text { Modèle } \\
\text { initial }\end{array}$ & $\begin{array}{c}\text { Modèle } \\
\text { respécifié }\end{array}$ \\
\hline \multirow{4}{*}{$\begin{array}{l}\text { Indices } \\
\text { absolus }\end{array}$} & $\mathrm{AGFI}$ & 0,9 & 0,795 & 0,802 \\
\cline { 2 - 5 } & GFI & 0,9 & 0,819 & 0,826 \\
\cline { 2 - 5 } & SRMR & $<0,05$ & 0,070 & 0,067 \\
\cline { 2 - 5 } & $\mathrm{RMSEA}$ & 0,08 & 0,038 & 0,037 \\
\cline { 2 - 5 } & $\mathrm{P}$ & $>0,05$ & 0,000 & 0,000 \\
\hline \multirow{2}{*}{$\begin{array}{l}\text { Indices } \\
\text { incrémentaux }\end{array}$} & $\mathrm{CFI}$ & 0,9 & 0,742 & 0,800 \\
\cline { 2 - 5 } $\begin{array}{l}\text { Indices de } \\
\text { parcimonie }\end{array}$ & $\mathrm{NFI}$ & 0,9 & 0,740 & 0,790 \\
\cline { 2 - 5 } & $\mathrm{Khi} / \mathrm{ddl}$ & 5 & 1,635 & 1,613 \\
\hline \multirow{2}{*}{ CAIC } & $\begin{array}{c}<\text { au CAIC du } \\
\text { modèle saturé }\end{array}$ & $\begin{array}{c}3382,412 \\
(10523,860)\end{array}$ & $\begin{array}{c}3255,326 \\
(10141,175)\end{array}$ \\
\hline
\end{tabular}

Tableau 6 - Indices d'ajustement du modèle de mesure globale Source : Données terrain

Certes, les indices d'ajustement de ce modèle sont en deçà des seuils standards admis. Cependant, au regard de la complexité du modèle, Roussel, Durrieu, Campoy et El Akremi (2002) préconisent de revoir le niveau des seuils à la baisse, voire à 0,80 pour les indices que sont AGFI, GFI, NFI et CFI. Ainsi, avons-nous retenu les indices d'ajustement par la méthode des moindres carrés généralisés (MCG). La fiabilité de chaque échelle est particulièrement satisfaisante (voir tableau 7) en raison du niveau satisfaisant des coefficients de Jöreskog (1971), dont la plus petite valeur est 0,898 (sécurité perçue). 


\begin{tabular}{|l|c|c|c|c|c|}
\hline Variables & $\begin{array}{c}\text { @ de } \\
\text { Jöreskog }\end{array}$ & Confiance & $\begin{array}{c}\text { Sécurité } \\
\text { perçue }\end{array}$ & $\begin{array}{c}\text { Facilité } \\
\text { d'utilisation }\end{array}$ & $\begin{array}{c}\text { Intention } \\
\text { d'utilisation }\end{array}$ \\
\hline Confiance & $\mathbf{0 , 9 0 9}$ & $\mathbf{0 , 6 6 8}$ & & & \\
\hline Sécurité perçue & $\mathbf{0 , 8 9 8}$ & 0,408 & $\mathbf{0 , 8 1 8}$ & & \\
\hline Facilité d'utilisation & $\mathbf{0 , 8 9 9}$ & 0,423 & 0,186 & $\mathbf{0 , 6 9 0}$ & \\
\hline Intention d'utilisation & $\mathbf{0 , 9 4 0}$ & 0,350 & 0,088 & 0,398 & $\mathbf{0 , 7 2 3}$ \\
\hline
\end{tabular}

Tableau 7 - Test de validité convergente - discriminante Source : Données terrain

Ces valeurs dépassent largement le seuil standard de 0,70 et permettent donc de dire que chaque échelle de mesure mobilisée dans ce travail de recherche est fiable. Par ailleurs, les valeurs de variance moyenne extraite (VME) sont aussi satisfaisantes, car elles sont toutes supérieures à 0,50 . Cela implique que chaque variable manifeste reflète effectivement sa variable latente associée et est moins corrélée avec son erreur de mesure (Fornell et Larcker, 1981; Hair, Black, Babin, Anderson et Tatham, 2006). Enfin, nous avons appréhendé la validité discriminante des mesures. La comparaison de chaque VME de variable latente, avec les carrés de corrélation entre la variable et chacune des autres, montre que la validité discriminante est acceptable. À cet effet, on aperçoit par exemple que la VME de la sécurité perçue $(0,818)$ est supérieure aux carrés de corrélation situés sur la même ligne et/ou sur la même colonne de la VME concernée.

\subsection{Le test d'hypothèse}

Après la validation de nos instruments de mesure, nous sommes passés au test d'hypothèse. Nos quatre hypothèses ont été testées et les résultats obtenus sont présentés dans le tableau suivant:

\begin{tabular}{|l|c|r|r|c|}
\hline \multirow{2}{*}{ Variables } & \multicolumn{4}{|c|}{ Liens directs } \\
\cline { 2 - 5 } & Estimate* & S.E.* & C.R.* & $\boldsymbol{P}^{*}$ \\
\hline Perception positive de la sécurité & $-0,03$ & 0,045 & $-0,653$ & 0,514 \\
\hline Perception négative de la sécurité & $-0,14$ & 0,049 & $-2,831$ & 0,005 \\
\hline Confiance & 0,125 & 0,054 & 2,324 & 0,02 \\
\hline Facilité d'utilisation & 0,136 & 0,054 & 2,536 & 0,011 \\
\hline
\end{tabular}

*Estimate : coefficient estimé; S.E : écart-type associé; C.R : t de Student; P : niveau de signification

Tableau 8 - Résultat du test d'hypothèse Source : Données terrain

\section{La confiance}

Les résultats suggèrent que la confiance influence positivement l'intention d'utilisation du paiement mobile. En effet, l'estimation est positive $(0,125$; influence positive) et $P=0,02$ (influence significative). Lorsque les individus ont confiance dans le paiement mobile, cela renforce leur intention d'utilisation. La confiance s'avère donc être un déterminant de l'intention d'utilisation du paiement mobile. De ce fait, nous pouvons affirmer que l'hypothèse 1 est validée :

- La confiance influence positivement lintention d'utilisation du paiement mobile en contexte de COVID-19.

\section{La sécurité perçue}

Les résultats obtenus montrent aussi que, sur les deux dimensions de la sécurité perçue, une seule influence l'intention d'utilisation du paiement mobile. En effet, pour la perception positive de la sécurité, nous observons une estimation négative $(-0,03$; influence négative) et $P=0,514$ (influence non significative). Quant à la perception négative, nous observons une estimation négative $(-0,14$; influence négative) et $P=0,005$ (influence significative).

La sécurité perçue avait été appréhendée selon deux perceptions : l'une négative et l'autre positive. Ainsi, lorsque l'individu perçoit négativement la sécurité, cela influence négativement son intention d'utilisation. Par contre, une perception positive de la 
sécurité n'influence pas significativement l'intention d'utiliser le paiement mobile. À partir des résultats obtenus, nous pouvons affirmer que l'hypothèse 2 est rejetée et que l'hypothèse 3 est validée :

- La perception positive de sécurité n'influence pas positivement lintention d'utilisation du paiement mobile en contexte de COVID-19;

- La perception négative de sécurité influence négativement lintention d'utilisation du paiement mobile en contexte de COVID-19.

\section{La facilité d'utilisation}

Concernant la facilité d'utilisation, les résultats montrent qu'elle influence positivement l'intention d'utilisation du paiement mobile. Nous observons que l'estimation est positive ( 0,136 ; influence positive) et $P=0,011$ (influence significative). Plus les individus trouvent facile l'utilisation du paiement mobile, plus leur intention d'utilisation est forte. Nous pouvons donc dire que l'hypothèse 4 est validée :

- La facilité d'utilisation influence positivement l'intention d'utilisation du paiement mobile en contexte de COVID-19.

\section{Discussion}

Nos résultats montrent que la confiance influence positivement l'intention d'utilisation du paiement mobile. Elle est indispensable à l'établissement de transactions récurrentes. En effet, la confiance envers le produit ou le service proposé incite les consommateurs à demeurer dans une relation commerciale avec l'entreprise. Ainsi, plus le consommateur a confiance dans le paiement mobile, plus son intention de l'utiliser est renforcée. Dans plusieurs autres études, la confiance s'est avérée être un prédicteur de l'intention. En effet, selon les travaux de Lagace et Gassenheimer (1991), la confiance est une attitude qui mène un partenaire à s'engager avec quelqu'un malgré une possibilité éventuelle de perte qui serait subie en raison du comportement de cette personne. Morgan et Hunt (1994) pensent même que la confiance est un élément de médiation clé dans le succès des échanges relationnels. Doney et Cannon (1997) indiquent que la confiance du client à l'égard d'une entreprise ou d'un fournisseur est positivement liée à son intention d'interagir avec l'entreprise en question. Ces résultats corroborent les nôtres et montrent l'importance à accorder à l'établissement de la confiance dans les relations commerciales.

Concernant la sécurité perçue, nos résultats montrent que la perception positive de la sécurité n'a pas d'incidence significative sur l'intention d'utilisation du paiement mobile. Quant à la perception négative, elle influence négativement l'intention des individus à utiliser le paiement mobile. Ainsi, un consommateur qui perçoit l'offre comme non sécurisée sera plus hésitant à utiliser le paiement mobile. En revanche, lorsqu'il estime que l'offre est sécurisée, cela ne l'incite pas particulièrement à utiliser le paiement mobile. Nos résultats montrent que l'incidence de la sécurité perçue dépend de la perception (positive ou négative) du consommateur. Cette distinction n'avait pas été clairement établie dans la littérature, même si plusieurs auteurs insistent sur l'importance du risque de sécurité. Les travaux existants montrent l'influence positive ou négative, sans plus d'explications. Par exemple, Schierz et ses collègues (2010) montrent dans leurs travaux que la sécurité perçue a une forte influence sur l'intention d'utilisation du paiement mobile. Plusieurs auteurs (Bhatnagar, Misra et Rao, 2000; Miyazaki et Fernandez, 2001) affirment que la peur des transactions non sécurisées inhibe l'achat.

Concernant la facilité d'utilisation, nos résultats montrent que c'est une variable qui explique l'intention d'utilisation du paiement mobile. Plus l'utilisation est simple, plus l'intention d'utilisation est renforcée. Les individus qui trouvent simple le paiement mobile sont plus enclins à l'utiliser. Ces résultats rejoignent ceux de Venkatesh, Morris, Davis et Davis (2003), qui ont montré dans leurs travaux que l'utilité ainsi que l'effort attendu (facilité d'utilisation) sont fortement reliés à l'intention d'utilisation. Les résultats vont également dans le même sens que ceux de Davis (1989), pour qui la facilité d'utilisation manifestée par l'absence d'effort et de contrainte augmente la probabilité d'utilisation d'un système.

\section{Conclusion}

L'objectif de cet article était d'identifier les déterminants de l'utilisation du paiement mobile, puis d'analyser leur influence sur l'intention d'utilisation. Pour atteindre cet objectif, nous avons mené une étude empirique composée de deux phases: l'une exploratoire et l'autre confirmatoire. La phase exploratoire s'est faite auprès de 200 individus, 
tandis que la phase confirmatoire s'est faite auprès de 250 individus choisis par convenance. À partir d'une modélisation par équations structurelles, nous avons testé nos quatre hypothèses de départ. Ainsi, les hypothèses 1,3 et 4 ont été validées. Quant à l'hypothèse 2, elle a été rejetée.

Même si la présente recherche permet de donner un début d'explication à la controverse sur l'influence des déterminants de l'intention d'utilisation du paiement mobile, surtout concernant la sécurité perçue (positive ou négative), elle est susceptible d'être améliorée sur divers plans. En effet, d'autres études peuvent être menées pour mieux élucider pourquoi la perception positive de sécurité n'a pas d'impact positif sur l'intention d'utilisation. Il serait aussi judicieux de continuer l'exploration afin d'identifier d'autres déterminants de l'intention d'utilisation du paiement mobile. Dans cette perspective, une piste de recherche serait l'étude du rôle joué par l'environnement (les proches) dans l'intention d'utiliser le paiement mobile.

\section{RÉFÉRENCES}

Adams, D., Nelson, R. et Todd, P. (1992). Perceived usefulness, ease of use, and usage of information technology: A replication. MIS Quarterly, 16(2), 227-247. https://doi.org/10.2307/249577

Ajzen, I. et Fishbein, M. (1975). Belief, attitude, intention, and behavior: An introduction to theory and research. Reading, MA : Addison-Wesley.

Autorité de régulation des télécommunications de Côte d'Ivoire. (Mars 2020). Statistiques du marché des télécommunications en Côte d'Ivoire 4ime trimestre 2019 : 2020. Repéré à https://www.artci.ci/images/stories/pdf/rapport_activite/ rapport_statistique_4e_trim_2019.pdf

Banque centrale des États de l'Afrique de l'ouest. (2015). Instruction n008-05-2015 régissant les conditions et modalités d'exercice des activités des émetteurs de monnaie électronique dans les états membres de l'union monétaire ouest africaine (UMOA) : 2015.

Repéré à https://www.bceao.int/sites/default/files/2017-11/instruction_no008_05_2015_intranet.pdf

Baumüller, H. (2018). The little we know: An exploratory literature review on the utility of mobile phone-enabled services for smallholder farmers. Journal of International Development, 30(1), 134-154. https://doi.org/10.1002/jid.3314

Bhatnagar, A., Misra, S. et Rao, R. (2000). On risk, convenience, and Internet shopping behavior. Communications of the ACM, 43(11), 98-105. https://doi.org/10.1145/353360.353371

Branscomb, L. M. et Thomas, J. C. (1984). Ease of use: A system design challenge. IBM Systems Journal, 23(3), 224-235. https://doi.org/10.1147/sj.233.0224

Chaix, L. (2013). Le paiement mobile : modèles économiques et régulation financière. Revue d'économie financière, 112(4), $277-298$. https://doi.org/10.3917/ecofi.112.0277

Chaix, L. et Torre, D. (2015). Le double rôle du paiement mobile dans les pays en développement. Revue économique, 66(4), 703-727. https://doi.org/10.3917/reco.664.0703

Dabholkar, P. A. et Bagozzi, R. P. (2002). An attitudinal model of technology-based self-service: Moderating effects of consumer traits and situational factors. Journal of the Academy of Marketing Science, 30(3), 184-201. https://doi.org/10.1177/0092070302303001

Davis, F. (1989). Perceived usefulness, perceived ease of use, and user acceptance of information technology. MIS Quarterly, 13(3), 319-340. https://doi.org/10.2307/249008

Davis, F. D., Bagozzi, R. P. et Warshaw, P. R. (1989). User acceptance of computer technology: A comparison of two theoretical models. Management Science, 35(8), 982-1003. https://doi.org/10.1287/mnsc.35.8.982

Deshmukh, S. P., Naware, A. M. et Mumbai, N. (2014). Mobile money: M-payment system for India. International Journal of Computer Science and Information Technologies, 5(2), 2672-2675.

Doney, P. et Cannon, J. (1997). An examination of the nature of trust in buyer-seller relationships. Journal of Marketing, 61(2), 35-51. https://doi.org/10.2307/1251829

Dube, B. (2020). Rural online learning in the context of COVID-19 in South Africa: Evoking an inclusive education approach. Multidisciplinary Journal of Educational Research, 10(2), 135-157. https://doi.org/10.17583/remie.2020.5607 
Ettis, S. et Haddad, M. (2014, mars). Valeurs perçues de l'Internet banking et comportement : rôle modérateur de la sécurité perçue. International Journal of Economics \& Strategic Management of Business Process. Communication présentée à la $2^{\mathrm{e}}$ International Conference on Business, Economics, Marketing and Management Research, Sousse, Tunisie. Repéré à https://docplayer.fr/9833013-Valeurspercues-de-l-internet-banking-et-comportement-role-moderateur-de-la-securite-percue.html

Fornell, C. R. et Larcker, D. F. (1981). Evaluating structural equation models with unobservable variables and measurement error. Journal of Marketing Research, 18(1), 39-50. https://doi.org/10.1177/002224378101800104

Ganesan, S. (1994). Determinants of long-term orientation in buyer-seller relationships. Journal of Marketing, 58(2), 1-19. https://doi.org/10.2307/1252265

Ganesan, S. et Hess, R. (1997). Dimensions and levels of trust: Implications for commitment to a relationship. Marketing Letters, 8(4), 439-448. https://doi.org/10.1023/A:1007955514781

Gerbing, D. et Anderson, J. (1988). An updated paradigm for scale development incorporating unidimensionality and its assessment. Journal of Marketing Research, 25(2), 186-192. https://doi.org/10.2307/3172650

Gronroos, C. (1990). Service management and marketing: Managing the moments of truth in service competition. Lexington, MN : Lexington Books.

Gurviez, P. et Korchia, M. (2002). Proposition d'une échelle de mesure multidimensionnelle de la confiance dans la marque. Recherche et Applications en marketing, 17(3), 41-59. https://doi.org/10.1177/076737010201700304

Hair, J. F., Black, W. C., Babin, B. J., Anderson, R. E. et Tatham, R. L. (2006). Multivariate data analysis. Upper Saddle River, NJ: Pearson Prentice Hall.

Hess, J. S. (1995). Construction and assessment of a scale to measure consumer trust. Dans B. Stern et G. Zinkhan (dir.), Actes de l'American Marketing Association Educators Conference (p. 20-26). Chicago, IL: AMA.

Jöreskog, K. G. (1971). Statistical analysis of sets of congeneric tests. Psychometrika, 36, 109-133. https://doi.org/10.1007/BF02291393

Jung, J. H., Kwon, E. et Kim, D. H. (2020). Mobile payment service usage: U.S. consumers' motivations and intentions. Computers in Human Behavior Reports, 1, 100-108. https://doi.org/10.1016/j.chbr.2020.100008

Kalakota, R. et Whinston, A. (1997). Electronic Commerce: A Manager's Guide. Reading, MA: Addison-Wesley.

Kramer, R. M. et Tyler, T. R. (1996). Trust in organizations: Frontiers of theory and research. Thousand Oaks, CA: SAGE Publications.

Lagace, R.R. et Gassenheimer, J.B. (1991). An Exploratory Study of Trust and Suspicion toward Sales people: Scale Validation and Replication, American Marketing Association Proceedings (Winter), 121-127.

Le Roux, A., Chandon, J. L. et Strazzieri, A. (1997). Une analyse confirmatoire de la mesure dimplication durable PLA. Actes du 13e Congrès international de l'Association française du marketing, Toulouse, France. Repéré à https:/ /www.afm-marketing.org/fr/ system/files/publications/fichier_969.PDF

Lee, T. M. (2005). The impact of perceptions of interactivity on customer trust and transaction intentions in mobile commerce. Journal of Electronic Commerce Research, 6(3), 165-180. Repéré à http://web.csulb.edu/journals/jecr/issues/20053/paper1.pdf

Mallat, N., Rossi, M., Tuunainen, V. K. et Öörni, A. (2009). The impact of use situation and mobility on the acceptance of mobile ticketing services. Actes de la 39e Annual Hawaii International Conference on System Sciences (p. 42-52), Kauia, HI.

Miyazaki, A. et Fernandez, A. (2001). Consumer perceptions of privacy and security risks for online shopping. Journal of Consumer Affairs, 35(1), 27-44. https://doi.org/10.1111/j.1745-6606.2001.tb00101.x

Moorman, C., Zaltman, C. G. et Deshpande, R. (1992). Relationships between providers and users of market research: The dynamics of trust within and between organizations. Journal of Marketing Research, 29, 314-329.

https://doi.org/10.1177/002224379202900303

Morgan, R. et Hunt, S. (1994). The commitment-trust theory of relationship marketing. Journal of Marketing, 58(3), 20-38. https://doi.org/10.2307/1252308

Organisation mondiale de la santé. (2020). Coronavirus disease (COVID-19) situation report-132: 2020. Repéré à https:/ /www.who.int/ $\mathrm{docs} /$ default-source/coronaviruse/situation-reports/20200531-covid-19-sitrep-132.pdf?sfvrsn=d9c2eaef_2

Perrien, J. et Ricard, L. (1995). The meaning of a marketing relationship: A pilot study. Industrial Marketing Management, 24(1), $37-43$. https://doi.org/10.1016/0019-8501(94)00029-V 
Pousttchi, K. et Schurig, E. M. (2004). Assessment of today's mobile banking applications from the view of customer requirements. Dans DBLP (dir.), Actes de la 37e Annual Hawaii International Conference on System Sciences (p. 10), Big Island, HI.

Rempel, J. K., Holmes, J. G. et Zanna, M. P. (1985). Trust in close relationships. Journal of Personality and Social Psychology, 49(1), 95-112. https://doi.org/10.1037/0022-3514.49.1.95

Rossiter, J. R. (2002). The C-OAR-SE procedure for scale development in marketing. International Journal of Research in Marketing, 19(4), 305-335. Repéré à http://ro.uow.edu.au/buspapers/196

Roussel, P., Durrieu, F., Campoy, E. et El Akremi, A. (2002). Méthodes d'équations structurelles : recherche et applications en gestion. Paris, France : Economica.

Schierz, P. G., Schilke, O. et Wirtz, B. W. (2010). Understanding consumer acceptance of mobile payment services: An empirical analysis. Electronic Commerce Research and Applications, 9(3), 209-216. https://doi.org/10.1016/j.elerap.2009.07.005

Segars, A. et Grover, V. (1993). Re-examining perceived ease of use and usefulness: A confirmatory factor analysis. MIS Quarterly, 17(4), 517-525. https://doi.org/10.2307/249590

Shneiderman, B. (1987). Designing the user interface: Strategies for effective human-computer interaction. ACM SIGBIO Newsletter, 9(1). https://doi.org/10.1145/25065.950626

Sirdeshmukh, D., Singh, J. et Sabol, B. (2002). Consumer trust, value, and loyalty in relational exchanges. Journal of Marketing, 66(1), 15-37. https://doi.org/10.1509/jmkg.66.1.15.18449

Sridharan, S., Pramod, T. C. et Sunitha, N. R. (2014). Architecting an integrated framework, for utility payment services using automated teller machines. Actes de l'International Conference on Science Engineering and Management Research (p. 1-6), Chennai, Inde.

Swanson, E. B. (1982). Measuring user attitudes in MIS research: A review. OMEGA : International Journal of Management Science, 10(2), 157-165. https://doi.org/10.1016/0305-0483(82)90050-0

Sweeney, J. C. et Soutar, G. N. (2001). Consumer perceived value: The development of a multiple item scale. Journal of Retailing, 77(2), 203-220. https://doi.org/10.1016/S0022-4359(01)00041-0

Thompson, R., Higgins, C. et Howell, J. (1991). Personal computing: Toward a conceptual model of utilization. MIS Quarterly, 15(1), 125-143. https://doi.org/10.2307/249443

Tougan, U. P. et Théwis, A. (2020). COVID-19 et sécurité alimentaire en Afrique subsaharienne : implications et mesures proactives d'atténuation des risques de malnutrition et de famine. International Journal of Progressive Sciences and Technologies, 20(1), 172-193. Repéré à https://ijpsat.ijsht-journals.org/index.php/ijpsat/article/view/1751

Van der Heijden, H. (2002). Factors affecting the successful introduction of mobile payment systems. Actes de la $15 \mathrm{e}$ Electronic Commerce Conference eReality: Constructing the eEconomy (p. 430-443), Bled, Slovénie.

Venkatesh, V., Morris, M., Davis, G. et Davis, F. (2003). User acceptance of information technology: Toward a unified view. MIS Quarterly, 27(3), 425-478. https://doi.org/10.2307/30036540

Venkatesh, V., Thong, J. et Xu, X. (2012). Consumer acceptance and use of information technology: Extending the unified theory of acceptance and use of technology. MIS Quarterly, 36(1), 157-178. http://doi.org/10.2307/41410412

Wang, Y., Wang, Y., Lin, H. et Tang, T. (2003). Determinants of user acceptance of Internet banking: An empirical study. International Journal of Service Industry Management, 14(5), 501-519. https:/ /doi.org/10.1108/09564230310500192

Yang, S., Lu, Y., Gupta, S., Cao, Y. et Zhang, R. (2012). Mobile payment services adoption across time: An empirical study of the effect of behavioral beliefs, social influences, and personal traits. Computers in Human Behavior, 28(1), 129-142. https://doi.org/10.1016/j.chb.2011.08.019

Zhang, Y. et Ma, Z. F. (2020). Impact of the COVID-19 pandemic on mental health and quality of life among local residents in Liaoning province, China: A cross-sectional study. International Journal of Environmental Research and Public Health, 17(7), 2381. https://doi.org/10.3390/ijerph17072381 